\title{
Randomness and optimality in enhanced DNA ligation with crowding effects
}

\author{
Takaharu Y. Shiraki, ${ }^{1, *}$ Ken-ichiro Kamei $\odot,{ }^{2}$ and Yusuke T. Maeda ${ }^{1}$ \\ ${ }^{1}$ Department of Physics, Kyushu University, Motooka 744, Fukuoka 819-0395, Japan \\ ${ }^{2}$ Kyoto University, Institute for Integrated Cell-Material Sciences (iCeMS), Yoshida-Ushinomiyacho, Sakyo-ku, Kyoto 606-8501, Japan
}

(Received 14 December 2019; accepted 5 March 2020; published 23 March 2020)

\begin{abstract}
Enzymatic ligation is essential for the synthesis of long DNA. However, a number of ligated products exponentially decay as DNA synthesis proceeds randomly. The control of ligation randomness is important to suppress exponential decay and achieve efficient synthesis of long DNA. This study analyzes the randomness in sequential DNA ligations, termed quantitative polymerase chain reaction (qPCR)-based statistical analysis of randomness (qPCR-bSTAR), using a probability distribution of ligated DNA length. Exponential decay is suppressed in a polymer solution. Further, DNA ligation is found to be activated at optimal crowded conditions. The theoretical model of kinetic ligation suggests that intermolecular attraction due to molecular crowding is involved in the optimal balance of ligation speed and available ligase. Our findings indicate that crowding effects enhance the synthesis of long DNA with large amounts of genetic information retained.
\end{abstract}

DOI: 10.1103/PhysRevResearch.2.013360

\section{INTRODUCTION}

Genetic polymers, such as DNA or RNA, need to be elongated to store information in their sequences. To produce a long DNA polymer, sequential ligation of DNA fragments catalyzed by a ligase enzyme is essential for the repair of DNA breaks in the genome of living cells and the in vitro synthesis of the artificial genome [1-3]. Since end-to-end ligation in a test tube is a random process, new short fragments are connected from both ends to produce longer DNA, but the concentration of ligated polymers decays with the number of newly added monomers $[4,5]$. Further, since ligation randomness restricts the abundance of the polymerized product, the mechanism for synthesizing long genetic polymers in nature and the technology by which random enzymatic ligation occurs are fundamental questions that have long been discussed.

One scenario to drive the efficient synthesis of long DNA is the local increase of short DNA fragments using physical transport effects. DNA fragments that range in size from a few tens of base pairs (bp) to a few hundred are accumulated due to molecular transport and thermal convection under temperature gradients [6-9]. A local temperature gradient or solute concentration gradient induces the directed motion of DNA as a solute, and consequently the trapped DNA exhibits an exponential increase in the concentration $[10,11]$. This DNA trapping process is considered to compensate for the significant decrease in long DNA polymers.

The other relevant scenario is the effect of the coexisting polymer, known as molecular crowding [12]. When DNA

\footnotetext{
*shiraki@phys.kyushu-u.ac.jp

Published by the American Physical Society under the terms of the Creative Commons Attribution 4.0 International license. Further distribution of this work must maintain attribution to the author(s) and the published article's title, journal citation, and DOI.
}

coexists with large concentrations of polymers with smaller gyration radii, attractive interactions occur among DNA due to an excluded volume effect $[13,14]$. This molecular crowding has a wide range of effects on the structural changes and catalytic activity of protein enzymes [15-19] in terms of subdiffusive motion [20-23]. The interplay between molecular crowding and DNA ligation is important, but the lack of experimental methods to measure and control the randomness of DNA ligation is a major hindrance to revealing the physical mechanism for long DNA synthesis in crowded conditions.

In this study, we investigated the effects of molecular crowding on long DNA synthesis using end-to-end ligation of short DNA fragments with coexisting polymers (polyethylene glycol, PEG). To reveal the randomness in the ligation process, the concentration of ligated DNA products was measured using a quantitative polymerase chain reaction (PCR) (qPCR)-based method. This method quantifies the length distribution of ligated DNA products with the number of ligated joints per molecule. We found that the ligated DNA fragments exhibited a gradual exponential decay in the presence of crowding agents when the number of ligated joints increases, and the production of long DNA is consequently significantly enhanced with a smaller decay rate. We further revealed the optimal conditions of molecular crowding by changing the PEG concentration. The exponential decay increases and the ligation efficiency decreases due to the reduction of freely available ligase as the PEG concentration is larger than $10 \%$. The control of such ligation randomness may be useful for the synthesis of artificial genomes in vitro and to understand enzymatic reactions in crowded conditions.

\section{RESULTS}

\section{A. Analysis of randomness in enzymatic ligation}

We examined the enzymatic ligation of short DNA fragments under macromolecular crowding conditions. To analyze 

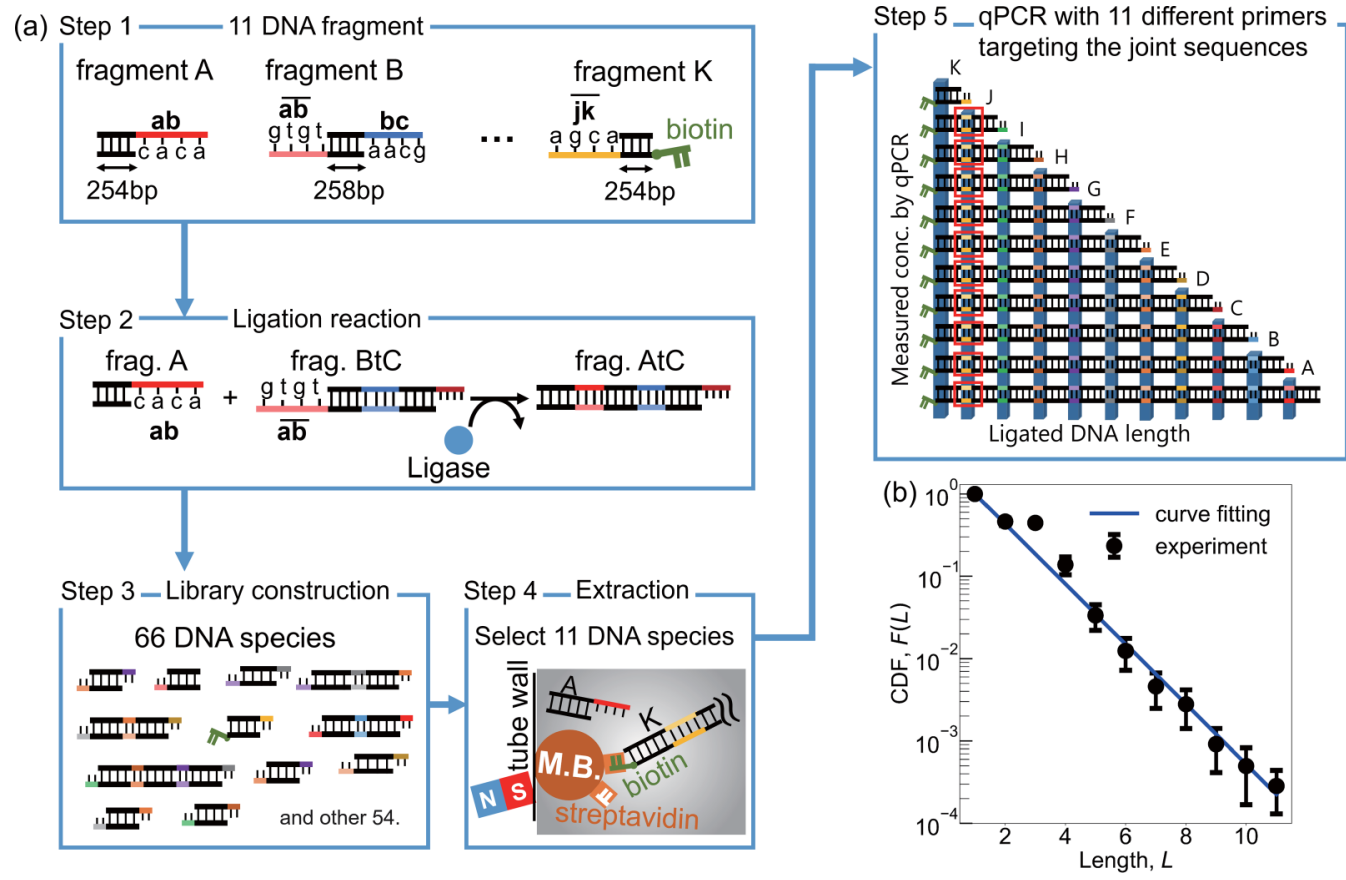

FIG. 1. Principle of the qPCR- $b$ STAR method. (a) Experimental procedure for qPCR- $b$ STAR. We prepared short DNA fragments (step 1) and performed DNA ligation (step 2). We obtained a DNA library of ligated products for the 66 species (step 3) and then selected a subpopulation of 11 DNA species by the pull-down method (step 4). M.B. stands for magnetic beads. In the final step, the qPCR analysis of the selected 11 DNA species was performed (step 5). qPCR- $b$ STAR quantifies the concentration of ligated DNA products as a cumulative distribution function (CDF) of DNA length. Using primers that detect sequences, including jk (red box), we measured the sum of concentrations of $\mathbf{J t K}, \ldots, \mathbf{B} \mathbf{t K}$, and AtK. (b) Experimentally measured CDF as a function of DNA length $L$ after ligation in a buffer solution. The experimental data fitted well with the exponential function $\exp (-0.84 L)$. Error bars represent the standard deviation of three independent experiments.

the mechanism regarding how long DNA strands are efficiently synthesized under crowding conditions, an analytical method that can analyze ligated DNA products based on their length and concentration is needed. This technical requirement motivates the development of a method for probing the probability distribution of ligated DNA with respect to its length, named qPCR-based statistical analysis of randomness (qPCR-bSTAR).

The experimental protocol of the qPCR- $b$ STAR is described in Fig. 1(a), as follows:

(i) First step: Short DNA fragments are synthesized by PCR amplification [top left in Fig. 1(a)]. These DNA fragments had 11 different sequences and were distinguished by labeling from $\mathbf{A}, \mathbf{B}, \ldots$, and $\mathbf{K}$. Their base pair (bp) numbers were all the same size and $258 \mathrm{bp}$. To create a ligated joint between DNA fragments, both ends had an overhang structure that was termed sticky ends. The recognition sites by restriction enzymes are indicated by letters, as $\overline{\mathrm{ab}}$ or bc ... ( $\overline{a b}$ is a complementary sequence of $a b$ ), and were added to their ends. Sticky ends were obtained as DNA substrates for the subsequent end-to-end ligation process.

(ii) Second step: DNA ligation was performed [top right in Fig. 1(a)]. DNA with sticky ends were ligated using Taq DNA ligase. For instance, the $\mathbf{B}$ and $\mathbf{C}$ fragments became a single DNA chain BtC after ligation, as bc and $\overline{b c}$ formed a hybridized joint.

(iii) Third step: The construction of a DNA library for 66 species. Each of the 11 DNA fragments has a unique sequence, and fragment $\mathbf{A}$ can bind only to fragment $\mathbf{B}$, and its product $\mathbf{A t B}$ only binds to fragment $\mathbf{C}$, and so on. Afterwards, the ligated DNA fragments, in alphabetical order [bottom right of Fig. 1(a)], have $11+10+\cdots+1=$ 66 types, which vary in sequence and length. The ordered sequence is represented by a simple description as with $\mathbf{X t Y}$, which indicates that the short DNA fragments are connected from the $\mathbf{X}$ fragment to the $\mathbf{Y}$ fragment. For instance, a DNA that includes a A-B-C-D sequence is referred to as AtD. Meanwhile, $\mathbf{K}-\mathbf{K}$ refers to the $\mathbf{K}$ fragment alone. In the following description, DNA length is the number of short DNA fragments that are contained per molecule.

(iv) Fourth step: The extraction of DNA for qPCR analysis. To determine the probability distribution function of ligated DNA in its length, DNA fragments with the $\mathbf{K}$ sequence at the end were extracted and their concentrations were measured by qPCR [bottom left in Fig. 1(a)]. We modified the blunt end of the $\mathbf{K}$ fragment with biotin and extracted 11 target DNA using the pull-down method, along with strong binding to streptavidin. The obtained DNA-magnetic beads were used for the qPCR analysis.

(v) Fifth step: qPCR with 11 different primers that target the sequences at the joint. The concentration of selected DNA products that contain the $\mathbf{K}$ fragment indicates the length distribution of ligated DNA. The number of unit DNA fragments $L(1 \leqslant L \leqslant 11)$ is proportional to the number of ligated joints per molecule (that is, $L-1$ ). 
(a)
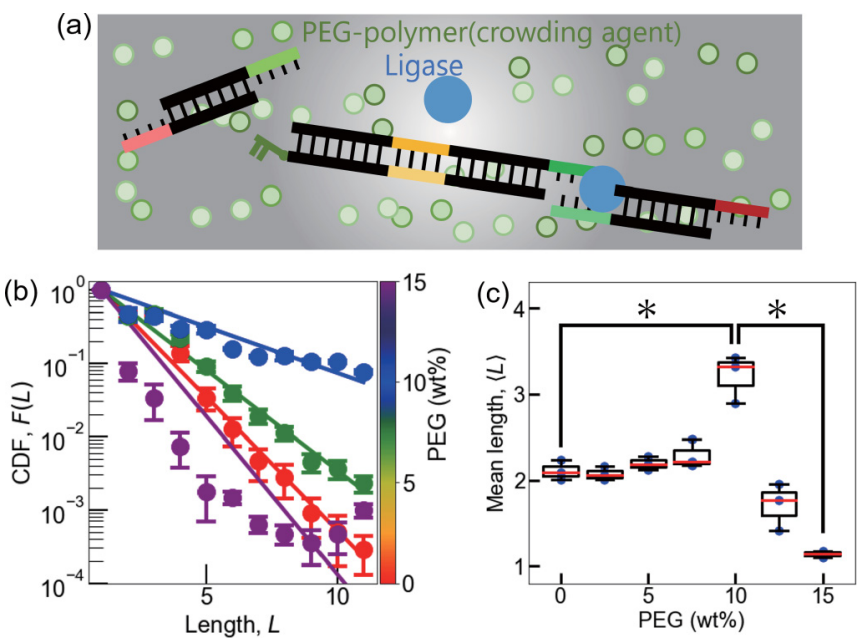

FIG. 2. The analysis of DNA ligation in polymer solutions using the qPCR-bSTAR method. (a) A schematic illustration of DNA ligation in a polymer solution with a crowding agent. The green particles represent crowding agents. We used polyethylene glycol (PEG) as the crowding agent. (b) Experimental results of CDF $F(L)$ of ligated DNA at various PEG concentrations. The color code represents the concentration of the crowding agent PEG, from $0 \%$ (red), $7.5 \%$ (green), and $10.0 \%$ (blue) to $15.0 \%$ (purple). The fitting curves are $F(L)=\exp (-\alpha L)$, with $\alpha=0.84$ in $0 \%$ PEG, $\alpha=0.63$ in $7.5 \%$ PEG, $\alpha=0.29$ in $10.0 \%$ PEG, and $\alpha=0.99$ in $15.0 \%$ PEG. Error bars represent the standard deviation of three independent experiments. (c) A box plot of the mean DNA length at various PEG concentrations. $* p<0.05$ (Mann-Whitney U-test) indicates a significant difference.

The concentration of DNA measured by qPCR analysis $C(L)$ is the cumulative concentration, $\sum_{n=L}^{11} c_{\mathrm{DNA}}(n)$. The normalized cumulative distribution function (CDF) of the ligated products, $F(L)=C(L) / C(1)$, was used to test whether $F(L)$ decays exponentially with DNA size. CDF $F(L)$ provides experimental insight into the randomness that underlies enzymatic ligation [Fig. 1(b)].

\section{B. Random DNA ligation with crowding effect}

We analyzed the ligation reaction in a buffer solution using the qPCR- $b$ STAR method. CDF $F(L)$ was plotted for DNA size $L$ [Fig. 1(c)] and exhibited exponential decay, $F(L)=$ $\exp (-\alpha L)$. The exponential decay of $F(L)$ shows that the ligation process is a random reaction that is not based on DNA length itself. Exponential decay was observed in the capillary electrophoresis analysis, although DNA fragments that were shorter than $L \leqslant 6$ were only detected [24].

The next question pertains to the mechanism regarding how exponential decay of $F(L)$ is suppressed due to physically relevant effects. While it has long been noted that molecular crowding of inert polymers increases enzyme activity [12], it is unclear whether ligation reactions occur randomly in a crowded environment [Fig. 2(a)]. Thus, we performed a qPCR- $b$ STAR assay for DNA ligation with various concentrations of polyethylene glycol 6000 (PEG) as a crowding agent from $2.5 \%$ to $15.0 \%$ (w/w). As shown in Fig. 2(b), $F(L)$ of the ligated DNA also exhibited exponential decay from $2.5 \%$ to $10.0 \%$ PEG, but the slope of the exponential decay was more gradual than the decay rate in the buffer solution $(0 \%$ PEG). This finding suggests that the ligation reaction occurred randomly even in the crowded environment, but the number of reaction products significantly increased. Moreover, as the PEG concentration reached $15.0 \%, F(L)$ exhibited stretched exponential decay, in which the tail of the length distribution was stretched slightly [Fig. 2(b), purple]. This suggests that for efficient synthesis of the longest product, DNA ligation has an optimal balance of enzymatic reaction and molecular crowding at approximately $10.0 \%$ PEG.

According to the exponential distribution function $F(L)$ at various PEG concentrations, the mean DNA length (the average number of ligations per molecule) $\langle L\rangle$ was analyzed. Figure 2(c) shows that $\langle L\rangle$ was increased by the addition of PEG and exhibited a peak at $10.0 \%$ PEG, but sharply decreased at higher concentrations. This optimal concentration of PEG implies that molecular crowding enhances the synthesis of longer DNA, but the effect that underlies the enhanced ligation was gradually suppressed in the overcrowded environment.

\section{Kinetics of DNA ligation}

The qPCR- $b$ STAR analysis allows determining that DNA ligation is random both in the buffer solution and crowded environments, but the mean length $\langle L\rangle$ can be affected by the concentration of PEG. However, the mechanism for an optimal concentration of PEG at enhanced DNA ligation is not clearly understood. To reveal the underlying mechanism, we measured the kinetic increase of ligated products with two pairs of $\mathbf{K}$ fragments and another DNA fragment that can be joined to the $\mathbf{K}$ fragment.

We first selected the $\mathbf{J}$ fragment of the same length, at $258 \mathrm{bp}$, to be the short DNA substrate. The rate of the enzymatic reaction in the presence of coexisting PEG was evaluated by measuring the ligated product of the $\mathbf{K}$ and $\mathbf{J}$ fragments. The ligation reaction began in an aqueous solution (0\% PEG), in which a small fraction of the solution was extracted every $1 \mathrm{~min}$, and the concentration of the product JtK was measured by qPCR [Fig. 3(a)]. The concentration of $\mathbf{J t K}$ increased linearly with time, and the slope of the reaction kinetics increased with the concentration of $\mathbf{J}$ [Fig. 3(a)]. This linear increase with time was also observed when PEG $10 \%$ was added [Fig. 3(b)]. However, when the PEG concentration exceeded 15\%, although the product $\mathbf{J t K}$ gradually increased linearly, the slope of the reaction curve became independent of the initial concentration of $\mathbf{J}$ [Fig. 3(c)]. Further, the ligation process proceeded at a constant rate regardless of the DNA concentration at $15 \%$ PEG, which represents the point at which the ligation reaction no longer depends on the number of substrates.

Next, we selected the AtJ fragment that is ligated to all 10 fragments from $\mathbf{A}$ to $\mathbf{J}$, which is 10 times longer than the $\mathbf{K}$ and $\mathbf{J}$ fragments. By analyzing the ligation kinetics of the $\mathbf{K}$ and AtJ, we found a linear increase in the ligation product AtK for both the 0\% [Fig. 3(d)] and 10.0\% [Fig. 3(e)] PEG, and the slope of the kinetics increased with an initial amount of the AtJ fragment. Interestingly, for the 15.0\% PEG, the slope of the kinetics was suppressed as the initial amount of the AtJ fragment increased [Fig. 3(f)]. These results indicate 

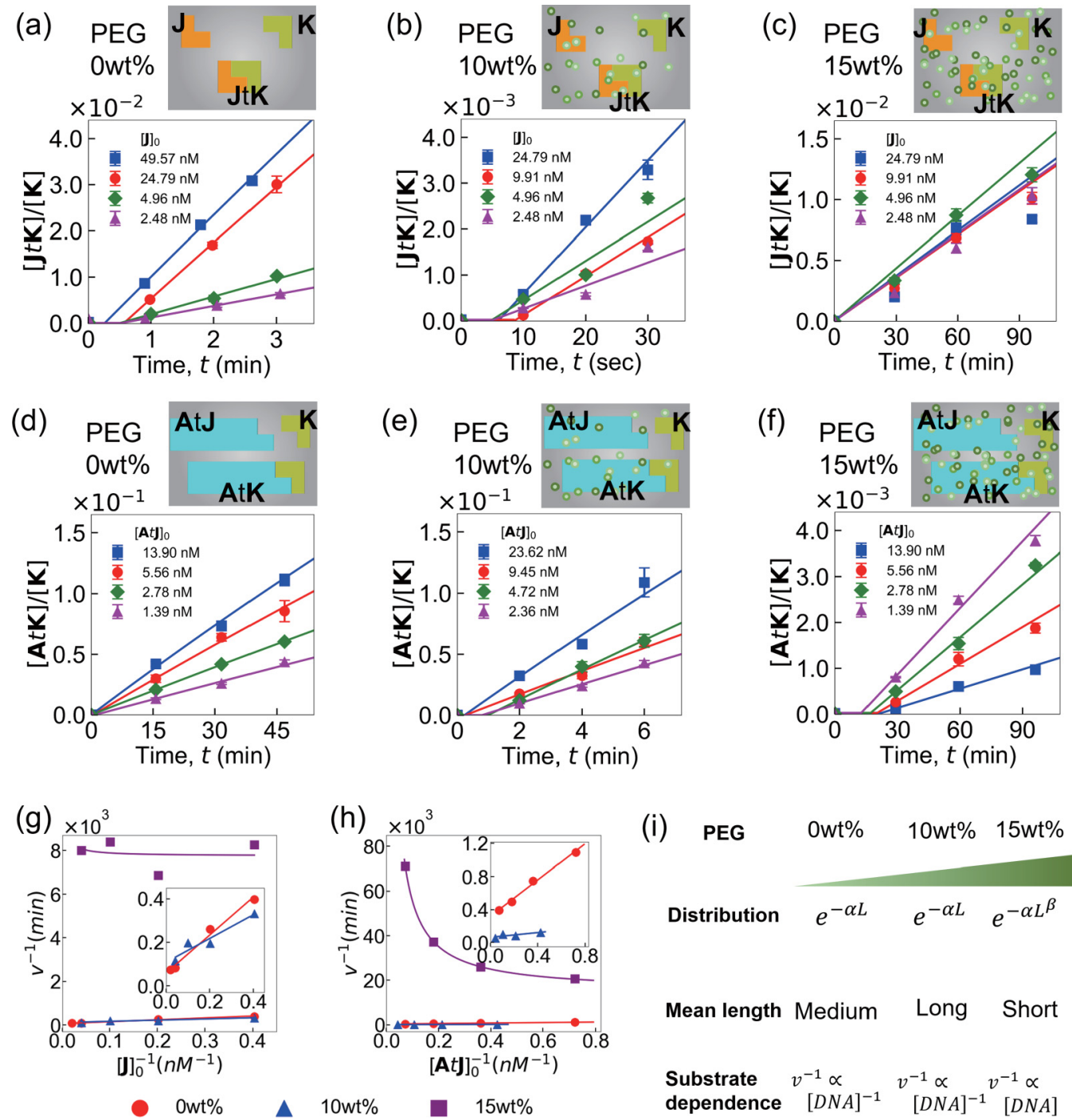

FIG. 3. Analysis of the kinetics of DNA ligation in crowding solutions using the qPCR-bSTAR method. (a)-(c) The relative amount of $\mathbf{J t K}$, which was synthesized by the ligation of $\mathbf{J}$ and $\mathbf{K}$, was plotted in time $t$ at various PEG concentrations. The color represents the initial concentration of the DNA substrate J. (a) 0.0\% PEG, (b) 10.0\% PEG, (c) 15.0\% PEG, (d) 0.0\% PEG, (e) 10.0\% PEG, (f) 15.0\% PEG. (d)-(f) The relative amount of AtK, which was synthesized by the ligation of At $\mathbf{J}$ and $\mathbf{K}$, was plotted in time $t$ at various PEG concentrations. The color represents the initial concentration of the DNA fragment AtJ. $(\mathrm{g}),(\mathrm{h})$ The Lineweaver-Burk plot of ligation reactions is shown. (g) DNA ligation of the $\mathbf{J}$ and $\mathbf{K}$ fragments. The fitting curve is a linear function. (h) DNA ligation of the short $\mathbf{K}$ fragment and long At $\mathbf{J}$ fragment. The fitting curve is a linear function at $0.0 \%$ and $10.0 \%$ PEG, but becomes an inverse linear function at $15.0 \%$ PEG. (i) Summary of the effects of molecular crowding on the length distribution and kinetics of DNA ligation. Randomness, mean length $\langle L\rangle$, and kinetic speed $v$ show distinct profiles at various PEG concentrations (from left to right: dilute, crowded, highly crowded regimes).

that the presence of the macromolecules confers size dependence of the substrate molecules in the DNA ligation reaction [Figs. 3(g)-3(i)].

\section{Kinetic model of ligation with crowding effect}

We then proposed a theoretical model in which two effects are considered to explain the concentration dependence of the production rate $v$ and the DNA concentrations of $\mathbf{J}$ and AtJ. The Michaelis-Menten model explains the kinetics of the enzymatic reaction by acting as an enzyme-substrate complex and an intermediate product. We extend this model to understand the optimal conditions for the enhanced DNA ligation process in crowding conditions.
Following conventional notations, we use $S_{i}$ in the modeling below as a symbol of DNA $i$, in which $i$ stands for an arbitrary sequence from all 66 DNA sequences, and $\left[S_{i}\right]$ is the concentration of DNA. The length of DNA $i$ is $L_{i}$. Presume that the ligation reaction primarily consists of three chemical processes: the first process is the hybridization of complementary DNA pairs, the second step is the complex formation of DNA pairs and the ligase enzyme, and the third step is to induce end-to-end DNA ligation at the DNA-ligase complex. From the reaction scheme shown in Fig. 4(a), when two DNA fragments are complementarily bound at the sticky ends, this paired DNA represented by $\left\{S_{i} S_{j}\right\}^{c m}$ becomes a substrate for a subsequent ligation reaction. Free ligase in a solution can bind to the DNA $\left\{S_{i} S_{j}\right\}^{\mathrm{cm}}$ and this complex 
(a)

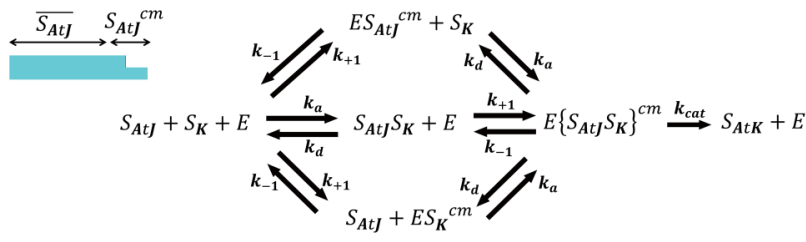

(b) Effect 1 : Stabilize DNA hybridization and binding of ligase to DNA [complementary hybridization]

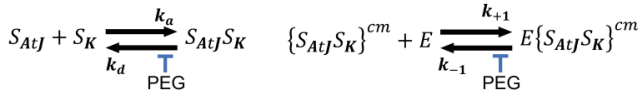

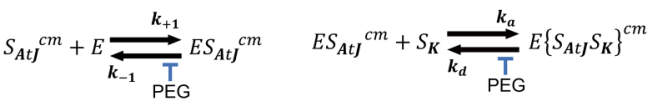

[non-complementary hybridization]

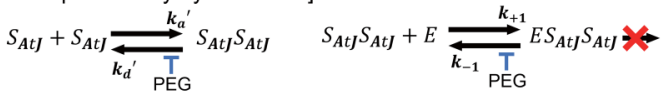

(c) Effect 2 : Non-specific binding of ligase to DNA

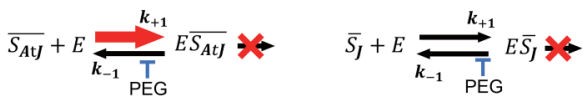

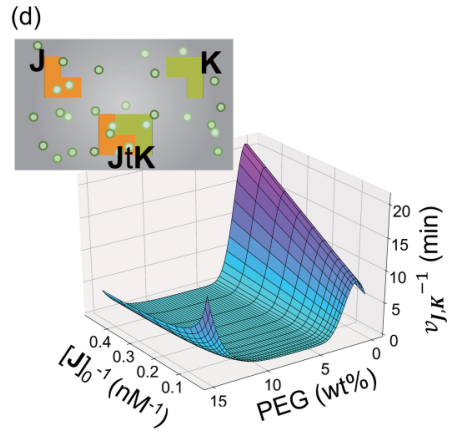

(e)

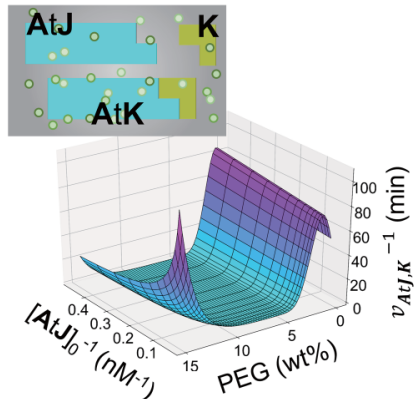

FIG. 4. A model of partial hybridization pathways in DNA ligation with molecular crowding. (a) A diagram of the ligation reaction using two DNA fragments, $S_{\mathrm{A} t \mathbf{J}}$ and $S_{\mathbf{K}}$, and ligase $E$. $S_{i}^{c m}$ is a part of $S_{i}$ that includes sticky ends. $\overline{S_{i}}$ is a part of $S_{i}$ that does not include sticky ends. $k_{a}$ is the binding constant of the complementary pair, $k_{d}$ is the dissociation constant of the hybridized pair, and $k_{+1}$ and $k_{-1}$ are the binding constant and the dissociation constant between DNA and ligase, respectively. (b),(c) Two distinct effects involved in DNA ligation with molecular crowding. (b) Effect 1: The stabilization of DNA hybridization by coexisting PEG molecules and the stabilization of partially hybridized DNA pairing. $k_{a}^{\prime}$ is the binding constant of the partially hybridized pair, and $k_{d}^{\prime}$ is the dissociation constant of the partially hybridized pair. (c) Effect 2: Nonspecific adsorption of ligase to DNA fragments in the presence of PEG. The red arrow indicates the length dependence of the nonspecific ligase-DNA binding, which is more easily adsorbed by longer DNA molecules. (d) Theoretical ligation speed for the ligation of the two short DNA fragments $\mathbf{K}$ and $\mathbf{J}$. The ligation speed is plotted for the initial DNA concentration $[\mathbf{J}]_{0}$ and the PEG concentration. (e) Theoretical ligation speed for the ligation of one short DNA fragment $\mathbf{K}$ and one long DNA fragment AtJ. The ligation speed is plotted as the initial concentration of the long DNA fragment $[\mathbf{A} t \mathbf{J}]_{0}$ and the PEG concentration.

is represented by $E\left\{S_{i} S_{j}\right\}^{c m}$. Given that the ligation reaction occurs as $S_{i}+S_{j} \rightarrow S_{i t j}$, the production rate is

$$
v_{i, j}=k_{\text {cat }}\left[E\left\{S_{i} S_{j}\right\}^{c m}\right],
$$

where $k_{\text {cat }}$ is the catalytic rate constant of the ligation reaction and $k_{\text {cat }}$ decreases exponentially as a function of PEG concentration $c_{\text {peg }}[24,25]$. The time evolution of $\left[E\left\{S_{i} S_{j}\right\}^{c m}\right]$ is then described by

$$
\begin{aligned}
\frac{d\left[E\left\{S_{i} S_{j}\right\}^{c m}\right]}{d t}= & k_{+1}[E]\left[S_{i} S_{j}\right]+k_{a}\left(\left[E S_{i}^{c m}\right]\left[S_{j}\right]+\left[S_{i}\right]\left[E S_{j}^{c m}\right]\right) \\
& -\left(k_{-1}+2 k_{d}+k_{\text {cat }}\right)\left[E\left\{S_{i} S_{j}\right\}^{c m}\right],
\end{aligned}
$$

where $E S_{i}^{c m}$ (or $E S_{j}^{c m}$ ) is a complex of sticky ends of DNA $S_{i}$ (or $S_{j}$ ), ligase $E, k_{a}$ is the kinetic constant for complementary binding of DNA at the sticky ends, $k_{d}$ is the kinetic constant for the dissociation of the sticky ends of DNA, $k_{+1}$ is the kinetic constant for the binding of ligase and DNA, and $k_{-1}$ is the kinetic constant of the ligase dissociation from the complexes. The stabilizing effects by molecular crowding induce the dependence of $k_{d}$ and $k_{-1}$ on $c_{\text {peg }}$ [24,25]. We assume that the catalytic rate constant $k_{\text {cat }}$ and other kinetic constants do not depend on the length or sequence of DNA, and thus each step of the DNA ligation process proceeds randomly regardless of the DNA species.
In the steady state at $d\left[E\left\{S_{i} S_{j}\right\}^{c m}\right] / d t=0, d\left[S_{i} S_{j}\right] / d t=$ $0, d\left[E S_{i}^{c m}\right] / d t=0$, and $d\left[E S_{j}^{c m}\right] / d t=0$, the following balance remains:

$$
\left[E\left\{S_{i} S_{j}\right\}^{c m}\right]=\Psi\left(c_{\mathrm{peg}}\right)^{-1}[E]\left[S_{i}\right]\left[S_{j}\right],
$$

where $\Psi\left(c_{\text {peg }}\right)$ is the dissociation constant of the ligase and two DNA fragments, as follows:

$$
\Psi\left(c_{\mathrm{peg}}\right)^{-1}=\frac{k_{+1} k_{a}}{k_{-1}+2 k_{d}+k_{\mathrm{cat}}}\left(\frac{1}{k_{d}}+\frac{1}{k_{-1}}\right) .
$$

Although these reaction schemes are ordinary ligation kinetics, to explain $v \propto\left[S_{i}\right]^{-1}$, as observed in Figs. 3(h) and 3(i), we need to consider additional pathways to the three ordinary steps. An additional effect that is considered is the formation of partial hybridization of a pair of DNA, in which the sequences of the sticky ends are not completely complementary [Fig. 4(b), effect 1]. The sticky ends have four bases for hybridization, but we assume that the DNA strands can cause partially hybridized pairing even in the presence of one or two base pair mismatches. Although the partial hybridization process is weaker in a buffer solution, molecular crowding due to the coexisting polymer stabilizes the partial hybridization process.

Another considered pathway is the nonspecific interaction of DNA and ligase. The ligase enzyme finds hybridized sticky 
ends and creates a new joint between two DNA fragments. We assume that the ligase protein is confined onto DNA fragments in a sequence-independent manner due to molecular crowding. In a dense PEG solution, molecular crowding induces attractive interactions between ligase (known as depletion force $[13,14])$. The longer DNA fragments have more space to attract ligase proteins and confine the active enzyme from the sticky ends [Fig. 4(c), effect 2]. This nonspecific binding onto DNA reduces the freely available enzyme in solution, which implicates that the effective concentration of ligase is decreased in the presence of long DNA in dense PEG solution.

These pathways induce the ligase and DNA form, in which the following three complexes do not contribute to the formation of ligated products. The first one is the complex $E S_{l} S_{m}$, in which ligase is bound to a DNA $S_{l} S_{m}$ that is incompletely joined at the ends of two DNAs, due to effect 1 . The second one is a complex, in which ligase binds to double-stranded sites other than sticky ends, such as $E \overline{S_{i}}, E \overline{S_{j}}$, and $E \overline{S_{i} S_{j}}$, due to effect 2 .

The amount of ligase is conserved in our experiment, and the total concentration of ligase $\left[E_{T}\right]$ is the summation of the concentration of the ligase-containing complex. By considering these pathways that appear in the crowded environment, $\left[E_{T}\right]$ is given by

$$
\left[E_{T}\right]=[E]+\Theta\left(\boldsymbol{S}, c_{\mathrm{peg}} ; \boldsymbol{L}\right)[E]+\sum_{(i, j) \in \mathbb{P}}\left[E\left\{S_{i} S_{j}\right\}^{c m}\right]
$$

and

$$
\begin{aligned}
\Theta\left(\boldsymbol{S}, c_{\mathrm{peg}} ; \boldsymbol{L}\right)[E]= & \sum_{(i, j) \in \mathbb{P}}\left[E \overline{S_{i} S_{j}}\right] \\
& +\sum_{(l, m) \in \mathbb{I}}\left[E S_{l} S_{m}\right]+\sum_{i}\left[E S_{i}\right],
\end{aligned}
$$

where $\sum_{(i, j) \in \mathbb{P}}$ is the sum of all possible DNA pairs with sticky end sequences that are complementary, $\sum_{(l, m) \in \mathbb{I}}$ is the sum of all DNA pairs with sticky ends that are complementary and cannot be ligated, and $\sum_{i}$ is the sum of all DNA. In Eq. (5), $\Theta\left(S, c_{\text {peg }} ; \boldsymbol{L}\right)$ represents the ratio of ligase trapped in DNA to the freely available ligase, $S$ is the concentration of all 66 DNA molecules $\left(\left[S_{\mathbf{A}}\right]\right.$, and $\left.\left[S_{\mathbf{A} t \mathbf{B}}\right], \ldots,\left[S_{\mathbf{A} t \mathbf{K}}\right]\right)$, and $\boldsymbol{L}$ are the lengths of all 66 DNA fragments $\left(L_{\mathbf{A}}, L_{\mathbf{A} t \mathbf{B}}, \ldots, L_{\mathbf{A} t \mathbf{K}}\right)$.

Finally, substituting Eqs. (3) and (6) into Eq. (5) (see details in [24]) and solving for [E] yields

$$
[E]=\frac{\left[E_{T}\right]}{1+\left(\Theta+\Psi^{-1} \sum_{(i, j) \in \mathbb{P}}\left[S_{i}\right]\left[S_{j}\right]\right)} .
$$

Further, Eqs. (1) and (7) thus attribute to the speed of the ligation reaction, in which

$$
v_{i, j}=\frac{k_{\mathrm{cat}}\left[E_{T}\right]\left[S_{i}\right]\left[S_{j}\right]}{\Psi(1+\Theta)+\sum_{(i, j) \in \mathbb{P}}\left[S_{i}\right]\left[S_{j}\right]} .
$$

To examine whether $v_{i, j}$ in Eq. (8) reproduces $v^{-1} \propto\left[S_{i}\right]$ at large PEG concentrations, we performed a numerical analysis at various $\mathrm{PEG}$ concentrations $c_{\mathrm{peg}}$ by changing the concentration of $\left[S_{i}\right]$, while maintaining $\left[S_{j}\right]$. The experimental results of Figs. 3(g) and 3(h) propose the following two cases of crowded ligation as model systems: the first is the ligation of the two short DNA fragments $i$ and $j$ that correspond to the $\mathbf{J}$ and $\mathbf{K}$ fragments, and the second is the ligation of long DNA and short DNA fragments that correspond to the AtJ and $\mathbf{K}$ fragments. Figure 4(d) [Fig. 4(e)] show the inverse speed of ligation $v_{\mathbf{J}, \mathbf{K}}^{-1}$ with the $\mathbf{J}$ fragment $\left(v_{\mathbf{A} t \mathbf{J}, \mathbf{K}}^{-1}\right)$ and the PEG concentration. We determine the following three distinct regimes in both surface plots: First, at the buffer solution $\left(c_{\text {peg }}=0\right), v^{-1}$ proportionally increased with $\left[S_{\mathbf{J}}\right]^{-1}$ (and $\left[S_{\mathbf{A} t \mathbf{J}}\right]^{-1}$ ). Second, $v^{-1}$ has a local minimum but is nearly constant even by changing $\left[S_{\mathbf{J}}\right]^{-1}$ (and $\left[S_{\mathbf{A} t \mathbf{J}}\right]^{-1}$ ) at intermediate PEG concentrations, including $10 \%$. Finally, $v^{-1}$ slows down when $\left[S_{\mathbf{J}}\right]^{-1}$ increases (and $\left[S_{\mathbf{A} t \mathbf{J}}\right]^{-1}$ increases) at a high concentration of PEG. In particular, the reaction of long and short DNA fragments in Fig. 4(e) evidently shows that the production of longer ligated DNA is suppressed by the addition of excessive amounts of substrate DNA, which is consistent with experimental results.

These distinct regimes can be explained using Eq. (8) as follows: the ligation of the short fragments $S=$ $\left(\left[S_{\mathbf{K}}\right],\left[S_{\mathbf{J}}\right],\left[S_{\mathbf{J} t \mathbf{K}}\right]\right)$, and $\mathbb{P}=((\mathbf{J}, \mathbf{K}))$, and the ligation of the short and long fragments $\boldsymbol{S}=\left(\left[S_{\mathbf{K}}\right],\left[S_{\mathbf{A} t \mathbf{J}}\right],\left[S_{\mathbf{A} t \mathbf{K}}\right]\right)$, and $\mathbb{P}=$ $((\mathbf{A t} \mathbf{J}, \mathbf{K}))$.

(i) DNA ligation in a buffer solution. At a dilute solution of small $c_{\text {peg }}$, the crowding effect is negligibly small and the ligase protein is rarely trapped in DNA. The fraction of the trapped ligases $\Theta \sim 0$ and $\Psi /\left[S_{\mathbf{K}}\right]=$ const. The DNA concentration depends on the ligation speed with $v^{-1} \sim 1+$ $\frac{\Psi}{\left[S_{\mathbf{K}}\right]\left[S_{\mathbf{J}}\right]} \sim\left[S_{\mathbf{J}}\right]^{-1}$ (and $\left[S_{\mathbf{A} t \mathbf{J}}\right]^{-1}$ ).

(2) Ligation of two short DNA in a polymer solution. Molecular crowding [effect 2 in Fig. 4(c)] induces nonspecific binding of ligase onto DNA in the PEG solution. At a higher concentration of PEG, the complementary-bound DNA complex is the first term of Eq. (6), which becomes dominant. This is because the matched complementary pairing is more stable than the partially hybridized DNA pairing. Further, nonspecific binding of ligase decreases the freely available enzymes from the solution, and the ratio of trapped enzymes becomes $\Theta \sim\left[S_{\mathbf{K}}\right]\left[S_{\mathbf{J}}\right]$. By considering this effect, the ligation of two short fragments proceeds at $v^{-1} \sim 1+\Psi \sim$ const.

(3) Ligation of long and short DNA in a polymer solution. Long DNA fragments also trap a larger amount of ligase by nonspecific binding, due to effect 2 in Fig. 4(c). For long DNA, the partially hybridized DNA due to effect 1 [Fig. 4(b)] is also presented and the longest DNA, such as $S_{\mathrm{A} t \mathbf{J}} S_{\mathrm{A} t \mathbf{J}}$, captures a large amount of ligase. The fraction of the trapped ligase is then approximated as $\Theta \sim\left[S_{\mathrm{A} t \mathbf{J}}\right]^{2}$. Thus, the ligation speed is inversely proportional to DNA concentration, as follows: $v^{-1} \sim 1+\Psi \frac{\left[S_{\mathrm{A} t \mathbf{J}}\right]^{2}}{\left[S_{\mathbf{K}}\right]\left[S_{\mathrm{A} t \mathbf{J}}\right]} \sim\left[S_{\mathrm{A} t \mathbf{J}}\right]$.

The mathematical analysis suggests that attractive but nonspecific binding due to molecular crowding causes the transition of ligation speeds with nontrivial length dependence.

\section{E. Optimal crowding effect for long DNA synthesis}

Finally, using the theoretical model based on Eq. (8), numerical simulations were conducted to verify the length distribution and the maximization of the concentration of long DNA fragments by ligation of many DNA strands. The ligation kinetics are described by the interplay of synthesis 

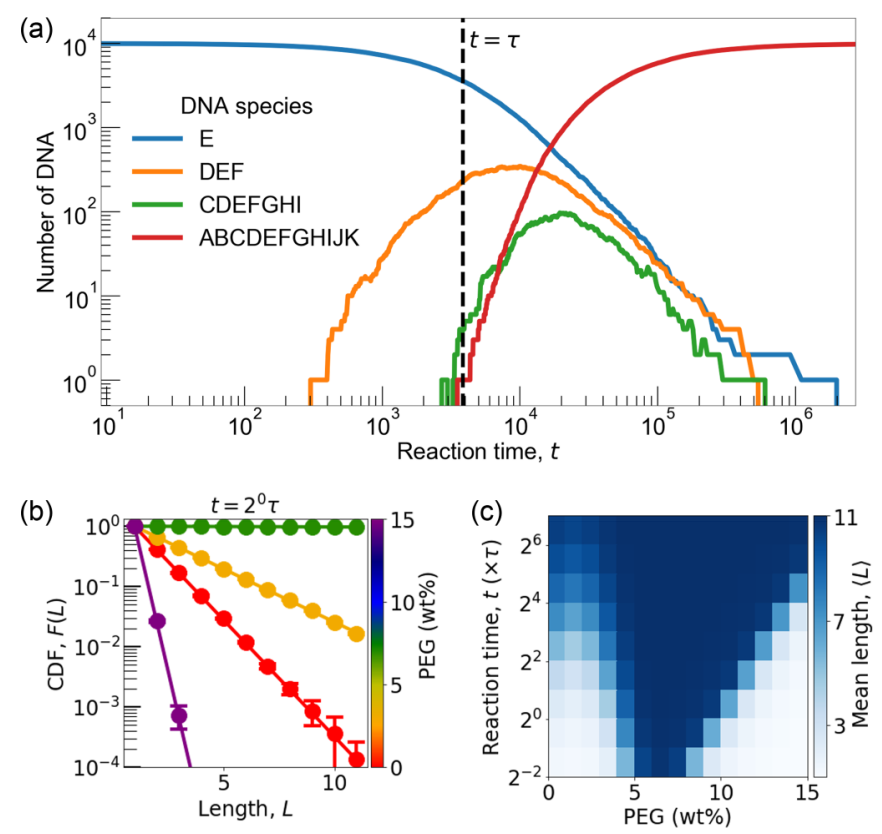

FIG. 5. Numerical analysis of stochastic ligation of 11 DNA species in crowded solutions. (a) Ligation kinetics of the number of ligated DNA products. The kinetics of four DNA species are plotted over time. We define the characteristic time $\tau$ as when the first longest DNA AtK was synthesized. (b) Normalized cumulative distribution function $F(L)$ with various concentrations of PEG. The color code represents the concentration of the crowding agent. The fitting curves are $F(L)=\exp (-\alpha L)$ with $\alpha=0.893$ in $0.0 \%$ PEG, $\alpha=0.407$ in $3.0 \%$ PEG, $\alpha=0.003$ in $7.0 \%$ PEG, and $\alpha=3.662$ in $15.0 \%$ PEG. (c) Mean length $\langle L\rangle$ in the concentration of the crowding agent and the reaction time $t$ (in units of $\tau$ ). The blue color represents the longer mean length.

and loss of $\left[S_{i}\right]$,

$$
\frac{d\left[S_{i}\right]}{d t}=\sum_{S_{n}+S_{m} \rightarrow S_{i}} v_{n, m}-\sum_{(i, l) \in \mathbb{P}} v_{i, l} .
$$

The first term represents the production of $S_{i}$ from all possible DNA pairing (the ligation of $S_{n}+S_{m} \rightarrow S_{i}$ ) and the second term represents a loss with substrates for other DNA reactions (the ligation of $S_{i}+S_{l} \rightarrow S_{k}$ ). The joining reaction of 11 types of DNA strands was numerically calculated using the Gillespie method, and the length distribution at the time when $t$ progressed for a certain time was plotted against the DNA strand length [Fig. 5(a)]. As the concentration of PEG increased, the slope of $F(L)$ decreased, but as the PEG concentration further increased, the slope of $F(L)$ became steep once again. Moreover, this model indicated that the concentration of the longest DNA [Fig. 5(b)] and the mean length of ligated DNA $\langle L\rangle$ [Fig. 5(c)] also exhibited a peak at intermediate PEG concentrations. On one hand, the crowding effect promotes joint formation and increases the rate of ligation per unit time. On the other hand, due to the large fraction of ligase, this effect would be confined to DNA at higher PEG concentrations, and the effective concentration of freely available enzyme decreases and the ligation reaction slows down. Such an interplay of enhanced binding and reduced enzyme concentration causes optimality for long DNA production.

\section{DISCUSSION}

In this study, we report enhanced ligation of DNA in a crowded polymer solution using qPCR-based statistical analysis. Thus, to avoid exponential decay due to random ligation, local concentration amplification has been proposed to cancel the decay by raising the initial concentration $[6,7,10,11]$. To increase the proportion of long DNA strands in a molecular population that is crowded with various kinds of polymer solutes [26], we found that molecular crowding by a coexisting polymer enhances the efficiency of the enzymatic reactionin particular, DNA ligation.

The stretched exponential decay of the ligated product was found under highly crowded conditions (15.0\% PEG). The longest DNA AtK is produced at concentrations higher than expected for exponential decay, which suggests a nonrandom reaction. However, this stretched exponential function was not found in the numerical simulation [Fig. 5(b)]. A possible explanation for this deviation is the length-dependent ligation process under crowded conditions. In Eq. (3), we assumed that the long DNA traps ligase more easily in crowded conditions [Fig. 4(c), effect 2], while ligase trapped in DNA does not exhibit catalytic activity. However, given that the trapped ligase retains catalytic functions, longer DNA fragments that store abundant ligases may be overlooked in the present model. Such length-dependent DNA ligation enhances the production of the longest DNA fragments in a nonrandom manner. Thus, the next question is to investigate the mechanism of enhanced synthesis of long DNA at the molecular level [27] under highly crowded conditions. It is also worth noting that the microscopic attraction between ligase and DNA is not experimentally detected in this study, although it is a plausible mechanism to explain optimal DNA ligation. The interaction of DNA-binding proteins with DNA has been extensively discussed [28], and an analysis will be described in detail in future studies.

Finally, crowded conditions exist in which high concentrations of proteins are present in living cells [29]. Whether enzymes react randomly or nonrandomly in these circumstances is poorly understood. The qPCR-bSTAR approach developed in this study may provide insight into intracellular enzyme reactions with crowding effects.

\section{MATERIALS AND METHODS}

\section{A. Synthesis of short DNA fragments}

Short DNA fragments that are used as substrates for ligation reactions were synthesized by polymerase chain reaction (PCR). DNA from the pUC19 plasmid was used as a template for the synthesis of 11 different nonoverlapping DNA fragments, and a KOD-plus-Neo polymerase (KOD-401, Toyobo) was used for DNA amplification. The resulting DNA fragments were $258 \mathrm{bp}$ long and were labeled alphabetically as $\mathbf{A}, \mathbf{B}, \ldots$, and $\mathbf{K}$. Oligonucleotide primers were designed using PRIMER3WEB [30-32] and were purchased from Eurofins Genomics. The sequences of the oligo primers that are used in this study are listed in the Supplemental Material [24]. 
Synthesized DNA was treated with a restriction enzyme and BstX1 (R0113L, New England BioLabs) at $37.0^{\circ} \mathrm{C}$ overnight. BstX1 recognition sites were added to the $5^{\prime}$ end of each primer. Purified DNA fragments have a sticky end at each side, except for those synthesized using the forward primer for the $\mathbf{A}$ fragments and the reverse primer for the $\mathbf{K}$ fragments. In addition, the reverse primer for the $\mathbf{K}$ fragments has a biotin at its $5^{\prime}$ end to capture the $5^{\prime}$ biotinylated $\mathbf{K}$ fragment for the pull-down step [Fig. 1(a), step 4].

\section{B. DNA ligation}

The final volume of the ligation reaction solution was $10 \mu \mathrm{L}$, whereby $7.27 \mathrm{ng}$ of 11 short DNA fragments and $20 \mathrm{U}$ thermostable Taq DNA ligase (M0208, New England Biolab) were mixed. The ligation reaction was performed at $25.0^{\circ} \mathrm{C}$ for $15 \mathrm{~h}$. Short DNA fragments were designed to be linked in alphabetical order, i.e., the $\mathbf{A}$ fragment was linked to the $\mathbf{B}$ fragment, and the $\mathbf{B}$ fragment binds to the $\mathbf{C}$ fragment.

\section{C. qPCR analysis}

The qPCR reaction volume was $10 \mu \mathrm{L}$. We mixed $5 \mu \mathrm{L}$ of $2 \times$ TB Green Premix Ex Taq II (RR820, TaKaRa) with $0.25 \mu \mathrm{L}$ of extracted DNA-magnetic beads, $0.5 \mu \mathrm{L}$ of the forward primer, $0.5 \mu \mathrm{L}$ of the reverse primer, and $3.75 \mu \mathrm{L}$ of
MilliQ water. The final concentration for each DNA primer was $400 \mathrm{nM}$. Thermal cycling for the qPCR analysis was performed using a real-time PCR system (CFX96, Bio-Rad) as follows: heating to $95.0^{\circ} \mathrm{C}$ for $30 \mathrm{~s}$, and 35 cycles of $95.0^{\circ} \mathrm{C}$ for $10 \mathrm{~s}$ and $60.0^{\circ} \mathrm{C}$ for $30 \mathrm{~s}$. The threshold cycle number $C_{q}$ was determined and the initial DNA concentration was calculated according to this value. Using pUC19 plasmid DNA as a template DNA of the known concentration, the $C_{q}$ value of each primer was plotted into a calibration curve of the $C_{q}$ value and DNA concentration.

To measure the ligation speed $v_{i, j}$, we mixed the $\mathbf{K}$ fragment (final concentration $48.6 \mathrm{pM}$ ) with either the $\mathbf{J}$ fragment or $\mathbf{A t} \mathbf{J}$ fragment at various concentrations. The ligation reaction was performed at $25.0^{\circ} \mathrm{C}$ with $6.4 \mathrm{U}$ thermostable Taq DNA ligase, and the volume of the reaction mixture was $4 \mu \mathrm{L}$. We selected time steps to observe linear increments of the relative amount of ligated DNA.

\section{ACKNOWLEDGMENTS}

This work was supported by a Grant-in-Aid for Scientific Research on Innovative Areas (Grants No. JP16H00805, No. JP17H05234, and No. JP18H05427) and Grant-in-Aid for Scientific Research (B) No. JP17KT0025 from MEXT, and Human Frontier Science Program Research Grant (Grant No. RGP0037/2015). We thank R. Sakai, K. Yoshimoto, and S. Terada for experimental assistance.
[1] B. Alberts, A. D. Johnson, J. Lewis, D. Morgan, M. Raff, K. Roberts, and P. Walter, Molecular Biology of the Cell (CRC, Boca Raton, FL, 2017).

[2] R. Phillips, J. Kondev, J. Theriot, and H. Garcia, Physical Biology of the Cell (CRC, Boca Raton, FL, 2012).

[3] C. A. Hutchison et al., Design and synthesis of a minimal bacterial genome, Science 351, aad6253 (2016).

[4] C. S. Reddy, A. Arinsteina, and E. Zussman, Polymerization kinetics under confinement, Polym. Chem. 2, 835 (2011).

[5] L. D. Landau and E. M. Lifshitz, Statistical Physics 1 (Butterworth-Heinemann, Oxford, UK, 1980).

[6] D. Braun and A. Libchaber, Trapping of DNA by Thermophoretic Depletion and Convection, Phys. Rev. Lett. 89, 188103 (2002).

[7] P. Baaske, F. M. Weinert, S. Duhr, K. H. Lemke, M. J. Russell, and D. Braun, Extreme accumulation of nucleotides in simulated hydrothermal pore systems, Proc. Natl. Acad. Sci. USA 104, 9346 (2007).

[8] C. B. Mast, S. Schink, U. Gerland, and D. Braun, Escalation of polymerization in a thermal gradient, Proc. Natl. Acad. Sci. USA 110, 8030 (2013).

[9] A. Priye, Y. Yu, Y. A. Hassan, and V. M. Ugaz, Synchronized chaotic targeting and acceleration of surface chemistry in prebiotic hydrothermal microenvironments, Proc. Natl. Acad. Sci. USA 114, 1275 (2017).

[10] Y. T. Maeda, A. Buguin, and A. Libchaber, Thermal Separation: Interplay Between the Soret Effect and Entropic Force Gradient, Phys. Rev. Lett. 107, 038301 (2011).
[11] Y. T. Maeda, T. Tlusty, and A. Libchaber, Effects of long DNA folding and small RNA stem-loop in thermophoresis, Proc. Natl. Acad. Sci. USA 109, 17972 (2012).

[12] B. H. Pheiffer and S. B. Zimmerman, Polymer-stimulated ligation: Enhanced blunt- or cohesive-end ligation of DNA or deoxyribooligonucleotides by T4 DNA ligase in polymer solutions, Nucl. Acid. Res. 11, 7853 (1983).

[13] D. Marenduzzo, K. Finan, and P. R. Cook, The depletion attraction: An underappreciated force driving cellular organization, $\mathrm{J}$. Cell Biol. 175, 681 (2006).

[14] S. Asakura and F. Oosawa, Interaction between particles suspended in solutions of macromolecules, J. Polym. Sci. B 33, 183 (1958).

[15] D. Kilburn, J. H. Roh, L. Guo, R. M. Briber, S. A. Woodson, and T. C. Jenkins, Molecular crowding stabilizes folded RNA structure by the excluded volume effect, J. Am. Chem. Soc. 132, 8690 (2010).

[16] J. S. Kim, V. Backman, and I. Szleifer, Crowding-Induced Structural Alterations of Random-Loop Chromosome Model, Phys. Rev. Lett. 106, 168102 (2011).

[17] H. Kang, P. A. Pincus, C. Hyeon, and D. Thirumalai, Effects of Macromolecular Crowding on the Collapse of Biopolymers, Phys. Rev. Lett. 114, 068303 (2015).

[18] B. Akabayov, S. R. Akabayov, S-J. Lee, G. Wagner, and C. C. Richardson, Impact of macromolecular crowding on DNA replication, Nat. Commun. 4, 1615 (2013).

[19] A. Singh and N. Singh, DNA melting in the presence of molecular crowders, Phys. Chem. Chem. Phys. 19, 19452 (2017). 
[20] J. Szymanski and M. Weiss, Elucidating the Origin of Anomalous Diffusion in Crowded Fluids, Phys. Rev. Lett. 103, 038102 (2009).

[21] I. M. Sokolov, Models of anomalous diffusion in crowded environments, Soft Matter 8, 9043 (2012).

[22] I. Budin and J. W. Szostak, Expanding roles for diverse physical phenomena during the origin of life, Annu. Rev. Biophys. 39, 245 (2010).

[23] D. Kestemont, M. Renders, P. Leonczak, M. Abramov, G. Schepers, V. B. Pinheiro, J. Rozenski, and P. Herdewijn, XNA ligation using T4 DNA ligase in crowding conditions, Chem. Commun. 54, 6408 (2018).

[24] See Supplemental Material at http://link.aps.org/supplemental/ 10.1103/PhysRevResearch.2.013360 for details of experimental methods and full theoretical details.

[25] L. Homchaudhuri, N. Sarma, and R. Swaminathan, Effect of crowding by dextrans and Ficolls on the rate of alkaline phosphatase-catalyzed hydrolysis: A size-dependent investigation, Biopolymers 83, 477 (2006).

[26] A. Blokhuis, D. Lacoste, P. Nghe, and L. Peliti, Selection Dynamics in Transient Compartmentalization, Phys. Rev. Lett. 120, 158101 (2017)
[27] I. Grossman-Haham, G. Rosenblum, T. Namani, and H. Hofmann, Slow domain reconfiguration causes power-law kinetics in a two-state enzyme, Proc. Natl. Acad. Sci. USA 115, 513 (2018).

[28] G.-W. Li, O. G. Berg, and J. Elf, Effects of macromolecular crowding and DNA looping on gene regulation kinetics, Nat. Phys. 5, 294 (2009).

[29] A. J. Boersma, I. S. Zuhorn, and B. Poolman, A sensor for quantification of macromolecular crowding in living cells, Nat. Methods 12, 227 (2015).

[30] A. Untergasser, I. Cutcutache, T. Koressaar, J. Ye, B. C. Faircloth, M. Remm, and S. G. Rozen, Primer3-New capabilities and interfaces, Nucl. Acids Res. 40, e115 (2012).

[31] T. Koressaar and M. Remm, Enhancements and modifications of primer design program Primer3, Bioinformatics 23, 1289 (2007).

[32] T. Koressaar, M. Lepamets, L. Kaplinski, K. Raime, R. Andreson, and M. Remm, Primer3-masker: Integrating masking of template sequence with primer design software, Bioinformatics 34, 1937 (2018). 